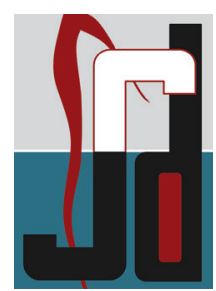

CASE SERIES

\title{
Compound Odontoma associated with Maxillary Canine: A Case Report
}

Eldhose K George $^{1}$, Sanju Lakshmanan ${ }^{2}$, Shilpa Chikkanna ${ }^{3}$, Eby Aluckal ${ }^{4}$, Abraham Kunnilathu ${ }^{5}$

ABSTRACT : Odontoma is a hamartomatous lesion of odontogenic origin involving both epithelial and mesenchymal tissues. Odontomas constitute about $22 \%$ of all odontogenic tumors of the jaws. They are usually asymptomatic but sometimes may interfere with the eruption of the associated tooth, leading to impaction or delayed eruption. This paper reports a case of management of the radiopaque mass which was associated with the impacted left maxillary canine. The radiopaque mass was surgically removed and confirmed as Compound Odontoma histopathologiocally

Keywords: Maxilla, Impacted teeth, Odontoma

\section{Introduction}

The term odontoma was first coined by Paul Broca in 1866 who defined it as "tumors formed by the overgrowth of transitory or complete dental tissues". Howard listed odontoma as the fourth category of supernumerary tooth, however not accepted universally. ${ }^{1}$ According to the recent literature, odontoma is considered to be a hamartomatous malformation rather than true neoplasm. ${ }^{2}$ Odontoma is a dysmorphic proliferation of both epithelial and mesenchymal components of dental tissues. Odontoma is neither capable of continuous growth nor complete differentiation into an organ of odontogenic origin and does not infiltrate into the surrounding tissues. It is supposed to originate from an extraneous bud of odontogenic cells from the dental lamina and is often associated with impacted teeth or retained deciduous teeth. Although definitive etiology are unknown, but possible hypotheses include infections, trauma, genetic factors and syndromic association.Cahn and Blum hypothesized that ameloblastic fibroma differentiates into odontoma whereas Eversole and Colleagues postulated that the individual mixed odontogenic tumors are incapable of further differentiation. ${ }^{3,4}$ According to the WHO classification, a complex odontoma is a malformation in which all the dental tissues are represented, individual tissues being mainly well formed but occurring in a more or less disorderly pattern; where as a compound odontoma is a malformation in which all the dental tissues are represented in a more orderly pattern than in the complex odontoma so that the lesion consists of many tooth like structures. ${ }^{5}$

Odontomas are usually asymptomatic, and they may be detected by chance on a routine radiograph (panoramic and/or intra-oral X-rays), or when they are large enough to cause a swelling of the jaw. Clinical signs suggestive of an odontoma include a retained deciduous tooth or an impacted tooth. ${ }^{6,7}$ Surgical removal is the treatment of choice of both complex and compound odontomas .7 Care should be taken, however, not to harm adjacent teeth and permanent tooth buds in children, while follow-up is essential for evaluation of further development of the permanent dentition at the removal location. Although the diagnosis of odontomas, in most cases, can be provisionally confirmed by radiographic examination, a histological study of the removed lesion must be performed to confirm the diagnosis.

\section{Case Report}

A 16 year old male patient reported to the department of Oral and Maxillofacial Surgery with a chief complaint of missing left maxillary canine. The family medical and dental histories were noncontributory. The patients as well as parents were unable to recall any trauma to the oral cavity or head and neck region. Extraoral examination revealed bilaterally symmetrical face and straight facial profile and missing left maxillary canine on intraoral examination. The panoramic radiograph revealed the presence of multiple tooth-like structures of different shapes and sizes in relation to the crown 
of the impacted permanent maxillary left canine which was situated apical to the maxillary left lateral incisor with extension into the maxillary sinus. They were surrounded by a well circumscribed radiolucent zone. Another tooth like structure was noticed between the maxillary central incisors. (Image1)Upon the clinical and radiographic findings, a provisional diagnosis of a compound odontoma was made and was decided to surgically enucleate the tumor along with impacted canine. Under local anesthesia, labial mucoperiosteal flap was elevated, the thin overlying bone was removed by chisel and rotary surgical burs to extract the multiple tooth-like structures known as denticles, and the size of the denticles varied from $4 \mathrm{~mm}$ to $10 \mathrm{~mm}$. (Image2) The flap was then closed with 3-0 black braided silk sutures (Image3) and the surgical specimen was submitted for histopathological examination. Postoperatively, the patient received systemic antibiotics, analgesics, and mouthwash for 7 days. There were no postoperative complications. On Follow up healing of the wound was satisfactory.

Histopathologiocally, the sections revealed miniature teeth containing dentin with normal arrangement of dentinal tubules, pulp, cementum, and periodontal ligament-like tissue. By correlating the clinical, radiological, and histopathological findings, a definite diagnosis of compound odontoma was made. (Image4)

\section{Discussion}

Several case series have documented that the majority of all odontomas were diagnosed in the first two decades of life. ${ }^{6}$ Although they may be discovered at any age, less than $10 \%$ are found in patients over 40 years old. Some studies have reported a correlation between patient age and the type of odontoma involved - compound lesions being apparently more frequent in younger patients, ${ }^{7}$ which is in agreement with our case. Discovery often occurs due to radiographic investigation for the cause of a non-erupted permanent or retained primary tooth. ${ }^{6,7}$ An impacted tooth is present in more than half of the cases. ${ }^{7}$ Iatrous et al., reported $80.7 \%$ odontomas in 26 cases were associated with impaction of permanent teeth. ${ }^{8}$ In our present case, the lesion was also associated with an impacted maxillary canine. When a panoramic radiograph was taken, an irregular radiopaque image with variations in contour and size, composed of multiple radiopacities corresponding to the so-called denticles, could be seen.
Most authors agree that these lesions effectively appear more often in the upper maxilla, though some sources make no distinction between the two maxillas., ${ }^{9} 10$ In our case the odontoma was located in the upper jaw, what is in accordance with the first theory. The reported tendency of odontomas to arise in the region of the incisors and canines ${ }^{10}$ is also confirmed in our case. Odontomas are more commonly found on the right side of the jaw ${ }^{11}$ where as we noticed on the left side of the maxilla. Considerable controversy exists over gender distribution. While some studies consider odontomas to be more common in females than in males, ${ }^{12,}{ }^{13}$ others consider these lesions to be similarly distributed between both genders. ${ }^{6,7}$ On the contrary, Iatrous et al. ${ }^{8}$ found a male predilection similar to our present case. Ameloblastic odontoma and Ameloblastic fibroodontoma bear great resemblance to the common odontoma, particularly on a radiograph, and thus it is suggested that all Odontomas should be sent to an oral pathologist for microscopic examination and definitive diagnosis. ${ }^{9}, 14,15$ Odontomas have been associated with trauma during primary dentition, as well as with inflammatory and infectious processes, hereditary anomalies (Gardner syndrome, Hermann's syndrome), odontoblastic hyperactivity and alteration in the genetic components responsible for controlling dental development. ${ }^{8,10}$ In our case no syndromes were evident and no episode of previous trauma was reported by the patient and family. In children, the impacted permanent teeth, depending on the age and on the tooth development, may be left to erupt spontaneously or guided to occlusion via orthodontic traction and if not possible, surgical extraction can be performed as we decided for our present case. In any case, follow-up is essential following odontoma excision. ${ }^{14,15}$

\section{Conclusion}

Early diagnosis and proper management of odontoma is necessary to prevent, later craniofacial developmental complications and allows adoption of a less complex and inexpensive treatment and ensures better prognosis. The thorough knowledge and an excellent evaluation of $\mathrm{X}$-ray documents are essentials to resolve adequately for each clinical case. The adoption of a conservative surgical approach is advisable, in order to preserve the dental tissues and obtain optimal tissue healing. A histological evaluation is necessary to confirm the diagnosis of odontoma. 


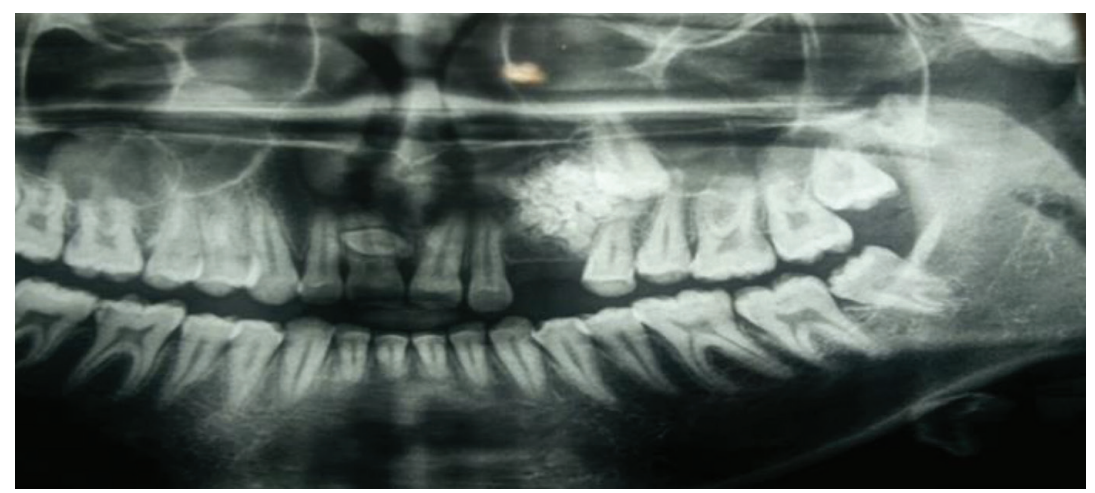

Fig 1: Multiple radiopaque teeth like structure associated with impacted left maxillary canine, mesodens/ tooth like structure

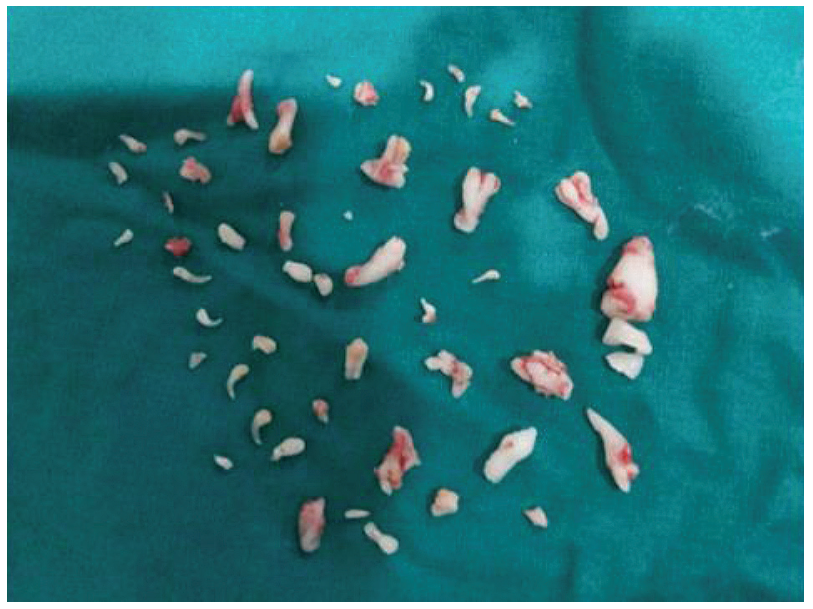

Fig 2: Multiple tooth like/denticles extracted

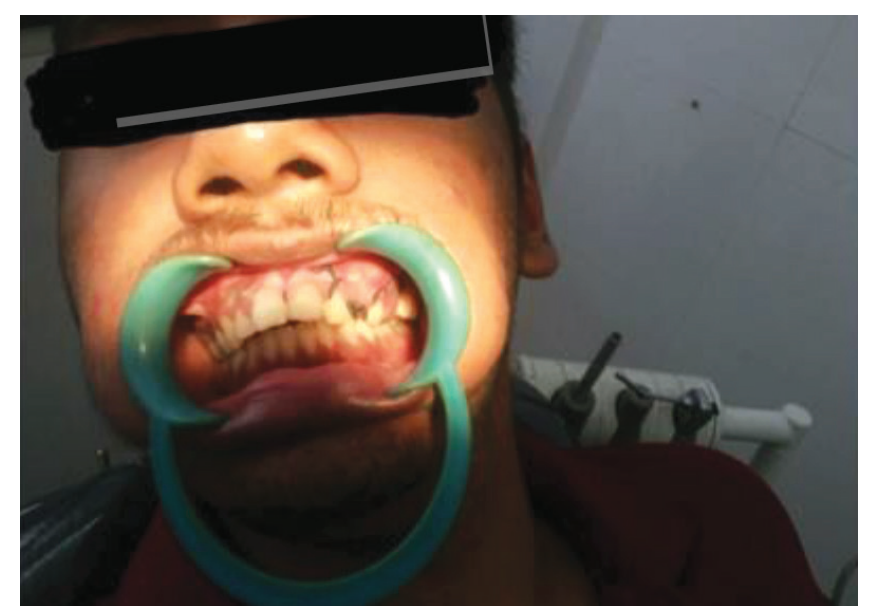

Fig 3: wound closure

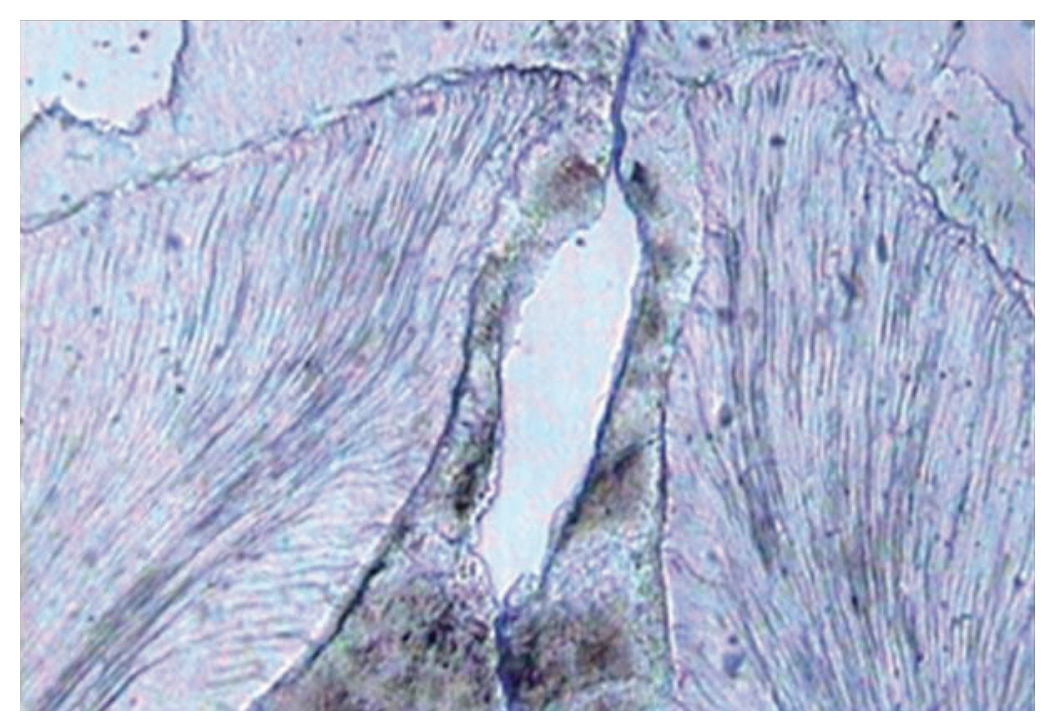

Fig 4: Ground section showing tooth-like structure made up of enamel, dentin and cementum (20X) 


\section{References}

1. Howard, R.D. (1967) The unerupted incisor. A study of the post operative eruptive history of incisors delayed in their eruption by supernumerary teeth. Dental Practitioner and Dental Record, 17, 332-41.

2. Budnick, S.D. (1976) Compound and complex odontoma. Oral Surgery, Oral Medicine, Oral Pathology, 42, 501-505. Doi: 10.1016/0030-4220(76)90297-8

3. Hawkins, P.L. and Sadeghi, E.M. (1986) Ameloblastic fibroodontoma: Report of case. J Oral Maxillofac Surg, 44, 10141019. Doi: 10.1016/S0278-2391(86) 80059-3

4. Slootweg, P.J. (1981) An analysis of the interrelationship of the mixed odontogenic tumors-Ameloblastic fibroma, ameloblastic fibro-odontoma, and the odontomas. Oral Surgery, Oral Medicine, Oral Pathology, 51, 266-276. Doi: $10.1016 / 0030-4220(81) 90056-6$

5. Takeda, Y. and Tomich, C.E. (2005) Ameloblastic fibroodontoma. In: World Health Organization Classification of Tumors (WHO). International Agency for Research on Cancer, IARC Press, Lyon, 309.

6. Nelson BL, Thompson LD. Compound odontoma. Head Neck Pathol. 2010; 4:290-1.

7. An S, An C, Choi K. Odontoma: a retrospective study of 73 cases. Imaging Sci Dent. 2012; 42:77-81.

8. Iatrous I, Vardas E, Theologie-Lygidakis N, Leventis M. A retrospective analysis of the characteristics, treatment and follow-up of 26 odontomas in Greek children. J Oral Sci. 2010; 52:439-47

9. 9. Baldawa R, Khante K, Kalburge J, Kasat V. Orthodontic management of an impacted maxillary incisor due to odontoma. Contemp Clin Dent. 2011; 2:37-40.

10. 10. Amado Cuesta S, Gargallo Albiol J, Berini Aytés L, Gay Escoda C. Revisión de 61 casos de odontoma. Presentación de un odontoma complejo erupcionado. Med Oral. 2003; 8:366-73.

11. 11.Shafer, Hine Lavy. Shafer's textbook of oral pathology. In: Rajendran R, Sivapathasundharam B, editors. Cysts and tumors of odontogenic origin. 6th ed. New Delhi: Elsevier; 2009. p. 287-90.

12. 12. Garcia-Consuegra L, Junquera LM, Albertos JM, Odontomas RO. A clinical-histological and retrospective epidemiological study of 46 cases. Med Oral. 2000;5:367-72.

13. 13. Hisatomi M, Asaumi J, Konouchi H, Honda Y, Wakasa T, Kishi K. A case of complex odontoma associated with an impacted lower deciduous second molar and analysis of the 107 odontomas. Oral Dis. 2002; 8:100-5.

14. 14. Lui JK, Hsiao CK, Chen HA, Tsai MY. Orthodontic correction of a mandibular first molar deeply impacted by an odontoma: a case report. Quintessence Int. 1997; 28:381-5.

15. 15. Frank CA. Treatment options for impacted teeth. J Am Dent Assoc. 2000; 131:623-32.

\section{Address of Correspondence}

Dr Eby Aluckal, MDS

Reader

Department of Public Health Dentistry

Annoor Dental College and Hospital,

Muvattupuzha, Ernakulam, Kerala, India - 686673

Cell : 919496097570

Email:draluckal@gmail.com

Authors:
${ }^{1}$ Professor and Head, Department of Oral and Maxillofacial
Surgery, Annoor Dental College and Hospital
${ }^{2}$ Reader, Department of Oral and Maxillofacial Surgery
Annoor Dental College and Hospital
${ }^{3}$ Senior Lecturer, Department of Oral and Maxillofacial Surgery
Annoor Dental College and Hospital
${ }^{4}$ Reader, Department of Public Health Dentistry, Annoor Dental
College and Hospital
${ }^{5}$ Research Associate, Western University, Canada

\section{Authors:}

illofacial

${ }^{2}$ Reader, Department of Oral and Maxillofacial Surgery ${ }^{3}$ Senior Lecturer, Department of Oral and Maxillofacial Surgery Reader, Department of Public Health Dentistry, Annoor Dental College and Hospital

\section{How to cite this article :}

Eldhose K George, Sanju Lakshmanan, Shilpa Chikkanna, Eby Aluckal, Abraham Kunnilathu. Compound Odontoma associated with Maxillary Canine: A Case Report. Journal of Scientific Dentistry, 2016;6(2):46-49

Source of Support : Nil, Conflicts of Interest : None declared 